\title{
Induced Remission of Metastatic Squamous Cell Carcinoma with an Immune Checkpoint Inhibitor in a Patient with Recessive Dystrophic Epidermolysis Bullosa
}

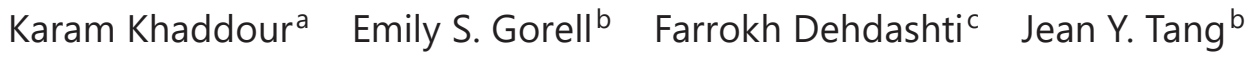 \\ George Ansstas ${ }^{a}$ \\ aWashington University in Saint Louis, Division of Medical Oncology, Saint Louis, MO, USA; \\ bStanford University School of Medicine, Department of Dermatology, Stanford, CA, USA; \\ 'Washington University in Saint Louis, Division of Nuclear Medicine, Department of \\ Radiology, Saint Louis, MO, USA
}

\section{Keywords}

Recessive dystrophic epidermolysis bullosa - Squamous cell carcinoma - Immune checkpoint inhibitor · Programmed death-1 inhibitor

\begin{abstract}
Recessive dystrophic epidermolysis bullosa (RDEB) is a genodermatosis that leads to skin fragility and chronic wound formation. Patients with RDEB are at risk for cutaneous squamous cell carcinoma (SCC) which is a major cause of morbidity and mortality in these patients. No standard of care exists for the treatment of SCC in this patient population and therapy is based on anecdotal reports and expert opinion. We report a 32-year-old man with RDEB with previously localized SCC who later developed metastatic SCC. He was started on cemiplimab (an immune checkpoint inhibitor) $350 \mathrm{mg}$ IV every 3 weeks. An objective radiological response was noted within 3 cycles. On 14 months follow-up, there was a durable response to treatment clinically and on imaging, without immune-related adverse events. To our knowledge, this is the first case report describing safe administration of immune checkpoint inhibitors in a patient with RDEB with objective and durable response of metastatic SCC. Larger case series and controlled clinical trials are needed to further investigate these medications in the RDEB population, given their high burden of aggressive and often lethal SCC.
\end{abstract}


Khaddour et al.: Checkpoint Inhibitor Use in Recessive Dystrophic Epidermolysis Bullosa

\section{Introduction}

Recessive dystrophic epidermolysis bullosa (RDEB) is a rare genetic skin disease caused by mutations in the gene COL7A1, leading to absent or defective type VII collagen, with skin fragility and resulting chronic wounds. These patients may develop extremely aggressive and often metastatic cutaneous squamous cell carcinoma (SCC). The risk for developing these lesions increases dramatically in the third decade; malignancy is often the cause of death [1]. Surgical treatment is the mainstay for localized tumors. There is no optimal therapy for advanced or metastatic SCC in this patient population given the partial short-lived response with conventional chemotherapy and the absence of controlled trials [2].

Immune checkpoint inhibitors block receptors in the tumor microenvironment responsible for immune suppression and malignancy progression. Cemiplimab is a monoclonal antibody that blocks programmed death-1 (PD-1) receptors leading to reactivation of the immune system against tumor cells. It was approved by the Food and Drug Administration in September 2018 to treat locally advanced and metastatic cutaneous SCC [3]. However, no reports currently exist in the literature describing its safety and efficacy in RDEB patients. We present a case of an RDEB patient with an objective durable response to cemiplimab therapy for metastatic SCC.

\section{Case Presentation}

A 32-year-old male presented with severe RDEB, confirmed via genetic testing (homozygous for c.8440C>T [p.R2814X] in exon 114 of COL7A1). He had a prior history of multiple SCCs on the lower back and right hand. Initial treatments of localized SCCs included numerous surgical resections. Recurrences were treated with topical imiquimod 5\%, 5-fluorouracil and calcipotriene followed by intradermal injection with 5-fluorouracil and methotrexate. However, the patient subsequently developed a large axillary lymph node and a new large fungating eroded nodule on the right forearm, positive for invasive SCC on shave biopsy.

The patient was treated several years prior with autologous keratinocyte skin grafts corrected with a retroviral vector locally to the upper back [4] and intradermal injections of autologous fibroblasts corrected with a lentiviral vector to the posterior neck and foot. SCC tissue samples and serum samples were negative for presence of these viruses.

Prior to initiation of therapy, positron emission tomography/computed tomography (PET/CT) with $\left[{ }^{18} \mathrm{~F}\right]$ fluorodeoxyglucose (FDG) revealed several foci of intense uptake along the right upper extremity including the right axilla, anterior right forearm, and dorsal and volar surfaces of the distal right forearm and hand (Fig. 1A), consistent with SCC sites. PET/ CT also revealed numerous foci of mildly increased FDG uptake associated with skin thickening throughout the body, likely related to RDEB.

The patient commenced cemiplimab $350 \mathrm{mg}$ IV every 3 weeks, with a single treatment of radiotherapy (total of $16 \mathrm{~Gy}$ ) to the right axilla and forearm. Two months after initiation of cemiplimab, repeat FDG-PET/CT showed marked metabolic response at the known sites of SCC in the right upper extremity and right axillary lymph nodes (Fig. 1B). There was no significant change in the numerous foci of mildly increased FDG uptake of the skin. No cutaneous lesions were visible on exam and no lymphadenopathy was apparent. Scans every 3 months were without evidence of SCC.

The patient reported mild nausea and fatigue, but denied any other side effects, including worsening RDEB wounds. There were no immune-related adverse events reported during or after initiation of immunotherapy. At the time of this report, he continues cemiplimab dosing every 3 weeks. The response has been sustained for 14 months.

\section{Karger's}




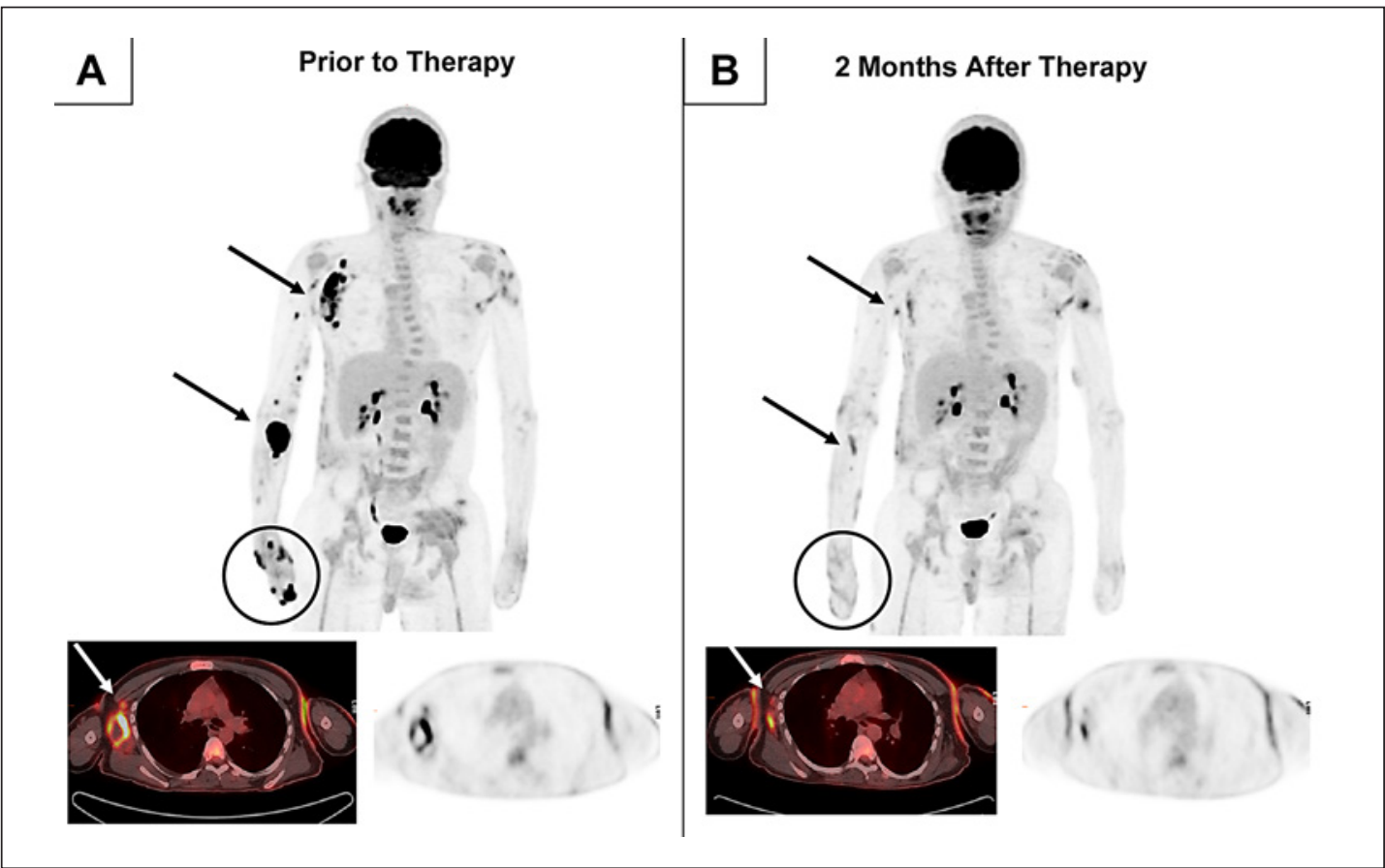

Fig. 1. PET/CT images prior to and following therapy with cemiplimab. A Anterior volume-rendered maximum activity-projection FDG-PET images show intense FDG uptake in the right upper extremity and right axillary lymph nodes on the pretherapy scan. The largest and most intense lesion corresponded to a FDGavid soft tissue mass in the anterior right forearm $(5.2 \times 4.6 \mathrm{~cm})$, with a maximum standardized uptake value $\left(S_{U} V_{\max }\right)$ of 33.3. A large conglomeration of right axillary lymph nodes with central photopenia $(6.7 \times 3.4$ $\mathrm{cm}, \mathrm{SUV}_{\max }$ of 22.8) is also present. Transaxial images consisting of fused PET/CT (left) and PET (right) at the level of axilla show intense FDG uptake within the right axillary lymph nodes. B PET/CT images following therapy demonstrated marked partial metabolic response. FDG uptake within the anterior right forearm, decreased in size to $1.2 \times 0.6 \mathrm{~cm}$ and FDG uptake ( $\mathrm{SUV}_{\max }$ of 5.7). The necrotic right axillary lymph nodes have decreased in size $(2.5 \times 0.9 \mathrm{~cm})$ and FDG uptake $\left(\operatorname{SUV}_{\max }\right.$ of 6$)$.

\section{Discussion}

SCC is a major cause of morbidity and mortality in RDEB patients. Median survival after SCC diagnosis is estimated at 2.4 years; over one third are estimated to develop metastatic disease [1]. SCC usually arises at chronic wounds and shows resistance and refractoriness to topical treatments as well as a pattern of recurrence after surgical resection. Chemotherapies have been described as a palliative approach in locally advanced and metastatic disease, with inconsistent outcomes [2]. Chemotherapies and targeted epidermal growth factor receptor inhibitor cetuximab have been described to be effective for metastatic SCC in RDEB patient [5]. However, these therapies typically incur a substantial risk for severe infections due to the need for indwelling vascular accesses, bone marrow suppression with neutropenia, and cutaneous side effects such as desquamation, mucositis, and severe (grade 3 to 4) acneiform reactions, which could be disastrous in patients with severe RDEB (Table 1) [2].

Cemiplimab is a monoclonal antibody that targets PD- 1 receptors on immune cells leading to augmentation of anti-tumor response. In an expansion cohort phase 1 study and a phase 2 single arm cohort, it has shown durable tumor responses in patients with cutaneous metastatic SCC without observed severe skin reactions, increased risk of neutropenia, or serious infections, which has led to its approval as a monotherapy of locally advanced and 
Khaddour et al.: Checkpoint Inhibitor Use in Recessive Dystrophic Epidermolysis Bullosa

Table 1. Comparison of conventional squamous cell carcinoma therapies and risks of severe cutaneous side effects

\begin{tabular}{ll}
\hline Therapy for squamous cell carcinoma & Side effects \\
\hline $\begin{array}{l}\text { Chemotherapies: alkylating agents, anthracyclines, antimetabolites, } \\
\text { antineoplastics [6] }\end{array}$ & - neutropenia \\
& - severe skin infections \\
& - severe septicemia \\
\hline $\begin{array}{l}\text { Epidermal growth factor receptor - tyrosine kinase inhibitors: cetuximab, } \\
\text { erlotinib, panitumumab [7] }\end{array}$ & - acneiform eruptions \\
& - skin irritation \\
\hline
\end{tabular}

Risk factors in patients with recessive dystrophic epidermolysis bullosa

Increased skin fragility

Large chronic wounds

Frequent wound infections, often with multi-drug-resistant bacteria

Chronic inflammatory state

metastatic SCC $[3,8]$. However, clinical trials of cemiplimab did not include RDEB patients. Given the significant risk of treatment complications associated with conventional chemotherapies and tyrosine kinase inhibitors, there is an unmet need for effective and safe therapies in metastatic SCC in patients with RDEB.

The loss of type VII collagen results in a fragile dermal-epidermal skin barrier causing development of chronically colonized and infected cutaneous wounds with multiple drugresistant bacteria that can often lead to sepsis [9]. This risk can be exacerbated when RDEB patients are treated with chemotherapies, which suppresses the bone marrow, and with tyrosine kinase inhibitors that can cause irritation of the skin barrier due to mucositis. The use of cemiplimab does not usually require indwelling vascular access. Furthermore, there have been only mild and minimal skin adverse events associated with the use of cemiplimab without observed bone marrow suppression.

Our patient demonstrated a sustained radiological response for 14 months, with minimal side effects and no immune-related adverse effects, which is promising. However, additional larger case studies and controlled trials are needed to confirm the safety and efficacy of cemiplimab in RDEB patients with cutaneous metastatic SCC.

\section{Conclusion}

To our knowledge, this is the first report of outcomes of treatment with a PD-1 inhibitor in a patient with metastatic SCC and RDEB. This report is important as patients with RDEB have high risk of metastatic SCC, which is a major cause of morbidity and mortality. No standard of care exists regarding these patients; therefore, we report the safety and efficacy with durable response (14 months) in one patient. This report should be interpreted with caution, as larger studies are needed to confirm the safety and efficacy of this approach.

\section{Statement of Ethics}

The patient has given his written informed consent to publish his case (including publication of images).

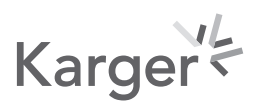




\section{Conflict of Interest Statement}

The authors have no competing interests to declare.

\section{Funding Sources}

No sources of funding were utilized in the preparation of this report.

\section{Author Contributions}

K.K. and E.S.G. contributed equally as first authors to the conceptualization, collection of data, and production of manuscript. F.D. provided the PET imaging with commentary and reviewed the work. J.Y.T. and G.A. supervised the whole work. All authors have read and approved the manuscript.

\section{References}

1 Fine JD, Johnson LB, Weiner M, Li KP, Suchindran C. Epidermolysis bullosa and the risk of life-threatening cancers: the National EB Registry experience, 1986-2006. J Am Acad Dermatol. 2009;60(2):203-11.

2 Mellerio JE, Robertson SJ, Bernardis C, Diem A, Fine JD, George R, et al. Management of cutaneous squamous cell carcinoma in patients with epidermolysis bullosa: best clinical practice guidelines. Br J Dermatol. 2016; 174(1):56-67.

3 Migden MR, Rischin D, Schmults CD, Guminski A, Hauschild A, Lewis KD, et al. PD-1 Blockade with Cemiplimab in Advanced Cutaneous Squamous-Cell Carcinoma. N Engl J Med. 2018 Jul 26;379(4):341-51.

4 Eichstadt S, Barriga M, Ponakala A, Teng C, Nguyen NT, Siprashvili Z, et al. Phase 1/2a clinical trial of genecorrected autologous cell therapy for recessive dystrophic epidermolysis bullosa. JCI Insight. 2019 Oct 3; 4(19):e130554.

5 Diociaiuti A, Steinke H, Nyström A, Schwieger-Briel A, Meiss F, Pfannenberg C, et al. EGFR inhibition for metastasized cutaneous squamous cell carcinoma in dystrophic epidermolysis bullosa. Orphanet J Rare Dis. 2019 Dec 3;14(1):278.

6 Lentz SR, Raish RJ, Orlowski EP, Marion JM. Squamous cell carcinoma in epidermolysis bullosa. Treatment with systemic chemotherapy. Cancer. 1990;66(6):1276-8.

7 Arnold AW, Bruckner-Tuderman L, Zuger C, Itin PH. Cetuximab therapy of metastasizing cutaneous squamous cell carcinoma in a patient with severe recessive dystrophic epidermolysis bullosa. Dermatology (Basel). 2009;219(1):80-3.

8 Migden MR, Khushalani NI, Chang ALS, Lewis KD, Schmults CD, Hernandez-Aya L, et al. Cemiplimab in locally advanced cutaneous squamous cell carcinoma: results from an open-label, phase 2, single-arm trial. Lancet Oncol. 2020 Feb;21(2):294-305.

9 Denyer J, Pillay E. Best practice guidelines for skin and wound care in epidermolysis bullosa. International consensus. http://www.woundsinternational.com/media/issues/623/files/content_10609.pdf

\section{Karger' ${ }^{\prime}=$}

